\title{
Concise Commentary: Penny Wise and Pound Foolish-Why SEMS Makes More Sense When Treating Post-anastomotic Biliary Strictures
}

\author{
Tawfik Khoury ${ }^{1,2} \cdot$ Manoj Kumar $^{3} \cdot$ Meir Mizrahi $^{4}$ \\ Published online: 25 September 2019 \\ ○) Springer Science+Business Media, LLC, part of Springer Nature 2019
}

Anastomotic biliary stricture (AS) is one of the most common complications following orthotopic liver transplant (LT) [1]. Most can be managed endoscopically, with only a few requiring reoperation/retransplantation. Endoscopic balloon dilation with placement of a plastic (polyethylene) or metal stent is successful in the majority. Plastic biliary stents are convenient, relatively inexpensive, and easy-toreplace. Nevertheless, plastic stents are prone to occlusion, necessitating frequent replacement and/or insertion of multiple side-by-side stents. These multiple ERCP sessions are accompanied by greater healthcare expenditures and enhanced risk. Fully covered self-expandable metallic stent (FCSEMS), although it costs more initially, may overcome some of these issues as it expands to a greater diameter and requires fewer reinterventions.

In this issue of Digestive Disease and Sciences, Jang et al. [2] reported a cost-effectiveness analysis of FCSEMS use versus plastic stent use for the treatment of anastomotic biliary stricture post-LT. In their analysis, FCSEMS reduced costs $25 \%$ compared with plastic stents while achieving similar rates of stricture resolution and complications.

When choosing plastic biliary stents or FCSEMS for post-LT anastomotic biliary stricture treatment, several questions must be considered: (1) How many interventions are required? (2) What is the success rate for stricture resolution? (3) What is the ease of insertion and time required? (4) What are the complications? (5) What is the total cost? In a recent systematic meta-analysis by Visconti et al., FCSEMS

Meir Mizrahi

mmizrahi@health.southalabama.edu

1 Department of Gastroenterology, Galilee Medical Center, Nahariya, Israel

2 Faculty of Medicine in the Galilee, Bar-Ilan University, Safed, Israel

3 Beth Israel Deaconess Medical Center, Boston, MA, USA

4 Division of Gastroenterology, University of South Alabama College of Medicine, Mobile, AL, USA required fewer total number of ERCP procedures which translated into a lower overall cost per patient ( $\$ 8288.50$ vs. $\$ 18,580.00$ for FCSEMS and plastic stents, respectively $P<0.001)$ with overall comparable adverse events [3].

The mean number of ERCP sessions with stent insertion needed to achieve stricture resolution is $3-5$ for plastic stents and 2 for FCSEMS. The most important contributor to overall cost appeared to be the number of ERCP sessions required rather than the initial cost of the stent [4].

In the current study, as well, the overall cost to achieve stricture resolution was significantly lower with FCSEMS $(\$ 12,942)$ compared with plastic stents $(\$ 17,190)$ although the initial cost of the FCSEMS itself was nearly tenfold greater than that of plastic stents ( $\$ 2775$ vs. \$291). Nonetheless, when the use of FCSEMS was incorporated at the third ERCP (meaning after failing two ERCP with plastic stents), the total cost for stricture resolution was not different between the two groups $(\$ 18,141$ for FCSEMS vs. $\$ 17,190$ plastic stents). The authors thus recommend early (at second ERCP) placement of FCSEMS in order to achieve cost-effectiveness.

To place these data in context, Martins et al. reported that although FCSEMS lowers the number of procedures required for stricture resolution and subsequently overall cost, it was associated with a higher number of adverse events including ERCP-related acute pancreatitis (FCSEMS, 13.3\%; plastic stents, $2.1 \% ; P<.01)$ [5]. An additional potential complication of FCSEMS is stent migration. Park et al. reported a FCSEMS migration rate of $0-41 \%$ [6]. In the current study, stent migration was also higher in those receiving FCSEMS (FCSEMS, 32.2\%; plastic stents, 23.9\%; $p<0.05)$ although there was no difference in the rate of ERCP-related pancreatitis or cholangitis between stent types.

Techniques to mitigate FCSEMS migration include placement of double pigtail biliary stents within the FCSEMS as well as the use of anti-migration stent systems [6]. In the current study, stent migration was treated adequately in most patients by the stent-in-stent technique. 
In the prevalent cost reduction environment, it seems reasonable to place FCSEMS early during treatment of anastomotic biliary strictures, usually at the second ERCP. Whether FCSEMS should be placed at the initial ERCP remains an unanswered.

\section{Compliance with Ethical Standards}

Conflict of interest All authors declare that they have no conflict of interest.

\section{References}

1. Akamatsu N, Sugawara Y, Hashimoto D. Biliary reconstruction, its complications and management of biliary complications after adult liver transplantation: a systematic review of the incidence, risk factors and outcome. Transpl Int. 2011;24:379-392. https:// doi.org/10.1111/j.1432-2277.2010.01202.x.

2. Jang S, Stevens T, Lopez R, et al. Self-expandable metallic stent is more cost efficient than plastic stent in treating anastomotic biliary stricture. Dig Dis Sci. (Epub ahead of print). https://doi. org/10.1007/s10620-019-05665-9.

3. Visconti TAC, Bernardo WM, Moura DTH, et al. Metallic vs plastic stents to treat biliary stricture after liver transplantation: a systematic review and meta-analysis based on randomized trials. Endosc Int Open. 2018;6:E914-E923. https://doi. org/10.1055/a-0626-7048.

4. Kao D, Zepeda-Gomez S, Tandon P, Bain VG. Managing the postliver transplantation anastomotic biliary stricture: multiple plastic versus metal stents: a systematic review. Gastrointest Endosc. 2013;77:679-691. https://doi.org/10.1016/j.gie.2013.01.015.

5. Martins FP, De Paulo GA, Contini MLC, Ferrari AP. Metal versus plastic stents for anastomotic biliary strictures after liver transplantation: a randomized controlled trial. Gastrointest Endosc. 2018;87:131.e1-131.e13. https://doi.org/10.1016/j. gie.2017.04.013.

6. Park DH, Lee SS, Lee TH, et al. Anchoring flap versus flared end, fully covered self-expandable metal stents to prevent migration in patients with benign biliary strictures: a multicenter, prospective, comparative pilot study (with videos). Gastrointest Endosc. 2011;73:64-70. https://doi.org/10.1016/j.gie.2010.09.039.

Publisher's Note Springer Nature remains neutral with regard to jurisdictional claims in published maps and institutional affiliations. 